\title{
A Simplified Derivation and Analysis of Fourth Order Runge Kutta Method
}

\author{
Musa $\mathrm{H}$. \\ Department of Mathematics and \\ Computer Science \\ UMYU
}

\author{
Ibrahim Saidu \\ Faculty of Computer Science and \\ Information Technology \\ University Putra,Malaysia.
}

\author{
M. Y. Waziri \\ Faculty of Science, Department of \\ Mathematics \\ University Putra,Malaysia
}

\begin{abstract}
The derivation of fourth order Runge-Kutta method involves tedious computation of many unknowns and the detailed step by step derivation and analysis can hardly be found in many literatures. Due to the vital role played by the method in the field of computation and applied science/engineering, we simplify and further reduce the complexity of its derivation and analysis by exploring some possibly well-known works and propose a step by step derivation of the method. We have also shown the stability region graphically
\end{abstract}

Keywords: Fourth order Runge Kutta Method, Derivation, Stability Analysis

\section{INTRODUCTION}

Runge-Kutta formulas are among the oldest and best understood schemes in numerical analysis. However, despite the evolution of a vast and comprehensive body of knowledge, it continues to be a source of active research [7]. Runge-Kutta methods provide a popular way to solve the initial value problem for a system of ordinary differential equations [11]:

$$
y^{\prime}=f(x, y), a \leq x \leq b, y(a)=y_{0}
$$

with a given step length $\mathrm{h}$ through the interval $[a, b]$, successively producing approximations $y_{n}$ to $y_{n+1}$. We deal exclusively with the step by step derivation and the stability analysis of the fourth order Runge-Kutta Method. For a thorough coverage of the derivation and analysis the reader is referred to $[1,2,3,4,5]$

The paper has the following structure: section 2 presents mathematical formulation and derivation, Section 3 presents the analysis and section 4 presents the conclusion.

\section{MATHEMATICAL FORMULATION AND DERIVATION}

We begin by defining the function as in $[1,2,3,4,5$ and 6$]$

$$
y_{n+1}=y_{n}+h \phi(x, y, h)
$$

$$
\begin{aligned}
& \text { Where } \phi(x, y, h)=\sum_{i=1}^{s} b_{i} k_{i} \\
& \qquad \begin{array}{l}
k_{1}=f(x, y) \\
k_{i}=f\left(x+c_{i} h, y_{n}+h \sum_{j=1}^{i-1} a_{i j} k_{j}\right), \quad i=2,3, \ldots, i-1 \\
c_{i}=\sum_{j=1}^{i-1} a_{i j} \\
y_{n+1}=y_{n}+h\left(b_{1} k_{1}+b_{2} k_{2}+b_{3} k_{3}+b_{4} k_{4}\right) \\
k_{1}=f(x, y) \\
k_{2}=f\left(x+c_{2} h, y_{n}+h a_{21} k_{1}\right) \\
k_{3}=f\left(x+c_{3} h, y_{n}+h\left(a_{31} k_{1}+a_{32} k_{2}\right)\right) \\
k_{4}=f\left(x+c_{4} h, y_{n}+h\left(a_{41} k_{1}+a_{42} k_{2}+a_{43} k_{3}\right)\right)
\end{array}
\end{aligned}
$$

The functions are expanded using a Taylor series expansion for function of two variables. To get the unknowns, we use the fourth order coefficients of order 4

$$
\begin{aligned}
& \tau_{1}^{(1)}=\sum_{i} b_{i}-1 \\
& \tau_{1}^{(2)}=\sum_{i} b_{i} c_{i}-\frac{1}{2} \\
& \tau_{1}^{(3)}=\frac{1}{2} \sum_{i} b_{i} c_{i}^{2}-\frac{1}{6} \\
& \tau_{2}^{(3)}=\sum_{i j} b_{i} a_{i j} c_{j}-\frac{1}{6} \\
& \tau_{1}^{(4)}=\frac{1}{6} \sum_{i} b_{i} c_{i}^{3}-\frac{1}{24} \\
& \tau_{2}^{(2)}=\sum_{i j} b_{i} c_{i} a_{i j} c_{j}-\frac{1}{8} \\
& \tau_{3}^{(4)}=\frac{1}{2} \sum_{i j} b_{i} a_{i j} c_{j}^{2}-\frac{1}{24} \\
& \tau_{4}^{(4)}=\sum_{i j} b_{i} a_{i j} a_{j k} c_{k}-\frac{1}{24}
\end{aligned}
$$

Setting the coefficients to zero, we have

$b_{1}+b_{2}+b_{3}+b_{4}=1$ 


$$
\begin{aligned}
& b_{2} c_{2}+b_{3} c_{3}+b_{4} c_{4}=\frac{1}{2} \\
& b_{2} c_{2}^{2} b_{3} c_{3}^{2}+b_{4} c_{4}^{2}=\frac{1}{3} \\
& b_{3} c_{3} a_{32} c_{2}+b_{4} c_{4} a_{42} c_{2}+b_{4} c_{4} a_{43} c_{3}=\frac{1}{8} \\
& b_{3} a_{32} c_{2}^{2}+b_{4} a_{42} c_{2}^{2}+b_{4} a_{43} c_{3}^{2}=\frac{1}{12} \\
& b_{4} a_{43} a_{32} c_{2}=\frac{1}{24}
\end{aligned}
$$

We use the simplifying assumptions by Butcher:

$$
\sum_{i=1}^{s} b_{i} a_{i j}=b_{i}\left(1-c_{j}\right), \quad j=2,3,4
$$

Which affect the expression for $\tau_{2}^{(3)}, \tau_{3}^{(4)}$ and $\tau_{4}^{(4)}$. i.e.

$\tau_{2}^{(3)}=\tau_{1}^{(2)}-2 \tau_{1}^{(3)}$

$\tau_{3}^{(4)}=\tau_{1}^{(3)}-3 \tau_{1}^{(4)}$

$\tau_{4}^{(4)}=\tau_{1}^{(2)}-2 \tau_{1}^{(3)}-\tau_{2}^{(4)}$

Now using equation (9) for $j=2,3$ and 4 we have:

$$
\begin{aligned}
& b_{3} a_{32}+b_{4} a_{42}=b_{2}\left(1-c_{2}\right) \\
& b_{4} a_{43}=b_{3}\left(1-c_{3}\right) \\
& 0=b_{4}\left(1-c_{4}\right) \text { respectively. }
\end{aligned}
$$

Now when $j=4$ in (iii), $c_{4}=1$ and $b_{4} \neq 0$ for a four stage method.

We substitute $c_{4}=1$ in equations 2, 3 and 5 and solve for $b_{2}$, $b_{3}$ and $b_{4}$ simultaneously. Therefore equations 2,3 and 5 becomes

$$
\begin{aligned}
& b_{2} c_{2}+b_{3} c_{3}+b_{4}=\frac{1}{2} \\
& b_{2} c_{2}^{2} b_{3} c_{3}^{2}+b_{4}=\frac{1}{3} \\
& b_{2} c_{2}^{3}+b_{3} c_{3}^{3}+b_{4}=\frac{1}{4}
\end{aligned}
$$

Using crammer's rule, we first find the determinant of the coefficient matrix

$$
\begin{aligned}
& b_{3} a_{32} c_{2}+b_{4} a_{42} c_{2}+b_{4} a_{43} c_{3}=\frac{1}{6} \\
& b_{2} c_{2}^{3}+b_{3} c_{3}^{3}+b_{4} c_{4}^{3}=\frac{1}{4}
\end{aligned}
$$

$D=\left|\begin{array}{lll}c_{2} & c_{3} & 1 \\ c_{2}^{2} & c_{3}^{2} & 1 \\ c_{2}^{3} & c_{3}^{3} & 1\end{array}\right|=-c_{2} c_{3}\left(c_{2}-1\right)\left(c_{2}-c_{3}\right)\left(c_{3}-1\right)$

To solve for $b_{2}$

$D_{b_{2}}=\left|\begin{array}{ccc}\frac{1}{2} & c_{3} & 1 \\ \frac{1}{3} & c_{3}^{2} & 1 \\ \frac{1}{4} & c_{3}^{3} & 1\end{array}\right|=\frac{-c_{3}\left(c_{3}-1\right)\left(2 c_{3}-1\right)}{12}$

$\therefore b_{2}=\frac{D_{b_{2}}}{D}=\frac{-c_{3}\left(c_{3}-1\right)\left(2 c_{3}-1\right)}{12} \div-c_{2} c_{3}\left(c_{2}-1\right)\left(c_{2}-c_{3}\right)\left(c_{3}-1\right)=\frac{1-2 c_{3}}{12 c_{2}\left(1-c_{2}\right)\left(c_{3}-c_{2}\right)}$

To solve for $b_{3}$

$$
\begin{aligned}
& D_{b_{3}}=\left|\begin{array}{ccc}
c_{2} & \frac{1}{2} & 1 \\
c_{2}^{2} & \frac{1}{3} & 1 \\
c_{2}^{3} & \frac{1}{4} & 1
\end{array}\right|=\frac{c_{2}\left(c_{2}-1\right)\left(2 c_{2}-1\right)}{12} \\
& \therefore b_{3}=\frac{D_{b_{3}}}{D}=\frac{c_{2}\left(c_{2}-1\right)\left(2 c_{2}-1\right)}{12} \div-c_{2} c_{3}\left(c_{2}-1\right)\left(c_{2}-c_{3}\right)\left(c_{3}-1\right)=\frac{1-2 c_{2}}{12 c_{3}\left(c_{3}-c_{2}\right)\left(1-c_{3}\right)}
\end{aligned}
$$

To solve for $b_{4}$

$$
\begin{aligned}
& D_{b_{4}}=\left|\begin{array}{lll}
c_{2} & c_{3} & \frac{1}{2} \\
c_{2}^{2} & c_{3}^{2} & \frac{1}{3} \\
c_{2}^{3} & c_{3}^{3} & \frac{1}{4}
\end{array}\right|=\frac{-c_{2} c_{3}\left(c_{2}-c_{3}\right)\left(3-4 c_{2}-4 c_{3}+6 c_{2} c_{3}\right)}{12} \\
& \therefore b_{4}=\frac{D_{b_{4}}}{D}=\frac{-c_{2} c_{3}\left(c_{2}-c_{3}\right)\left(3-4 c_{2}-4 c_{3}+6 c_{2} c_{3}\right)}{12} \div-c_{2} c_{3}\left(c_{2}-1\right)\left(c_{2}-c_{3}\right)\left(c_{3}-1\right)=\frac{6 c_{2} c_{3}-4\left(c_{2}+c_{3}\right)+3}{12\left(1-c_{2}\right)\left(1-c_{3}\right)}
\end{aligned}
$$

Now to solve for $a_{43}$, we use equation (ii) i.e. when $\mathrm{j}=3$

Hence, we have

$$
\Rightarrow a_{43}=\frac{b_{3}\left(1-c_{3}\right)}{b_{4}}=\frac{1-2 c_{2}}{12 c_{3}\left(c_{3}-c_{2}\right)\left(1-c_{3}\right)} \times\left(1-c_{3}\right) \times \frac{12\left(1-c_{2}\right)\left(1-c_{3}\right)}{6 c_{2} c_{3}-4\left(c_{2}+c_{3}\right)+3}
$$




$$
=\frac{\left(1-c_{2}\right)\left(2 c_{2}-1\right)\left(1-c_{3}\right)}{c_{3}\left(c_{2}-c_{3}\right)\left(6 c_{2} c_{3}-4\left(c_{3}+c_{2}\right)\right)+3}
$$

To solve for $a_{32}$ and $a_{42}$, we use equations (i) (when j=2) and (8) i.e.

$$
\begin{aligned}
& a_{32}=\frac{1}{24 c_{2}} \times \frac{1}{b_{4}} \times \frac{1}{a_{43}}=\frac{1}{24 c_{2}} \times \frac{12\left(1-c_{2}\right)\left(1-c_{3}\right)}{6 c_{2} c_{3}-4\left(c_{3}+c_{2}\right)+3} \times \frac{c_{3}\left(c_{2}-c_{3}\right)\left(6 c_{2} c_{3}-4\left(c_{3}+c_{2}\right)+3\right)}{\left(1-c_{2}\right)\left(2 c_{2}-1\left(1-c_{3}\right)\right.} \\
& =\frac{c_{3}\left(c_{2}-c_{3}\right)}{2 c_{2}\left(2 c_{2}-1\right)}
\end{aligned}
$$

Substituting this value into (i), we have

$$
\begin{aligned}
a_{42} & =\frac{b_{2}\left(1-c_{2}\right)-b_{3} a_{32}}{b_{4}} \\
& =\left[\frac{1-2 c_{3}}{12 c_{2}\left(1-c_{2}\right)\left(c_{3}-c_{2}\right)} \times\left(1-c_{2}\right)-\frac{1-2 c_{2}}{12 c_{3}\left(1-c_{3}\right)\left(c_{3}-c_{2}\right)} \times \frac{c_{3}\left(c_{2}-c_{3}\right)}{2 c_{2}\left(2 c_{2}-1\right)}\right] \times \frac{12\left(1-c_{2}\right)\left(1-c_{3}\right)}{6 c_{2} c_{3}-4\left(c_{2}+c_{3}\right)+3} \\
& =\frac{\left(1-c_{2}\right)\left\{2\left(1-c_{3}\right)\left(1-2 c_{3}\right)-\left(c_{2}-c_{3}\right)\right\}}{\left.2 c_{2}\left(c_{2}-c_{3}\right)\left\{6 c_{2} c_{3}-4\left(c_{2}+c_{3}\right)+3\right)\right\}}
\end{aligned}
$$

This solution assumes that

$$
c_{2} \neq 0,1, \quad c_{3} \neq 0,1, \quad c_{2} \neq c_{3}, \quad c_{2} \neq \frac{1}{2}
$$

We choose two free parameters $c_{2}=\frac{1}{3}$ and $c_{3}=\frac{2}{3}$

Substituting these values into $b_{4}, b_{3}$ and $b_{2}$ we have:

$$
\begin{aligned}
& b_{4}=\frac{6\left(\frac{1}{3}\right)\left(\frac{2}{3}\right)-4\left(\frac{2}{3}+\frac{1}{3}\right)+3}{12\left(1-\frac{1}{3}\right)\left(1-\frac{2}{3}\right)}=\frac{\frac{4}{3}-1}{\frac{8}{3}}=\frac{1}{8} \\
& b_{3}=\frac{1-2\left(\frac{1}{3}\right)}{12\left(\frac{2}{3}\right)\left(1-\frac{2}{3}\right)\left(\frac{2}{3}-\frac{1}{3}\right)}=\frac{\frac{1}{8}}{\frac{8}{9}}=\frac{3}{8} \\
& b_{2}=\frac{1-2\left(\frac{2}{3}\right)}{12\left(\frac{1}{3}\right)\left(1-\frac{1}{3}\right)\left(\frac{1}{3}-\frac{2}{3}\right)}=\frac{\frac{-1}{3}}{\frac{-8}{9}}=\frac{3}{8}
\end{aligned}
$$

Using equation (1)

$$
\begin{aligned}
b_{1}+b_{2}+b_{3}+b_{4}=1 \\
\Rightarrow b_{1}=1-b_{2}-b_{3}-b_{4} \\
=1-\frac{3}{8}-\frac{3}{8}-\frac{1}{8}=\frac{1}{8}
\end{aligned}
$$

Also $c_{2}=a_{21}=\frac{1}{3}$

Using equation (ii) (when $\mathrm{j}=3$ ),

$$
b_{4} a_{43}=b_{3}\left(1-c_{3}\right)
$$

$$
\begin{aligned}
& b_{3} a_{32}+b_{4} a_{42}=b_{2}\left(1-c_{2}\right) \\
& b_{4} a_{43} a_{32} c_{2}=\frac{1}{24}
\end{aligned}
$$

From equation (8) above,

$$
a_{43}=\frac{b_{3}\left(1-c_{3}\right)}{b_{4}}=\frac{3}{8} \times\left(1-\frac{2}{3}\right) \frac{8}{1}=1
$$

Also

$$
\begin{aligned}
a_{42}= & \frac{\left(1-c_{2}\right)\left\{2\left(1-c_{3}\right)\left(1-2 c_{3}\right)-\left(c_{2}-c_{3}\right)\right\}}{\left.2 c_{2}\left(c_{2}-c_{3}\right)\left\{6 c_{2} c_{3}-4\left(c_{2}+c_{3}\right)+3\right)\right\}} \\
= & \frac{\left(1-\frac{1}{3}\right)\left\{2\left(1-\frac{2}{3}\right)\left(1-2 \times \frac{2}{3}\right)-\left(\frac{1}{3}-\frac{2}{3}\right)\right\}}{\left.2 \times \frac{1}{3}\left(\frac{1}{3}-\frac{2}{3}\right)\left\{6 \times \frac{1}{3} \times \frac{2}{3}-4\left(\frac{1}{3}+\frac{2}{3}\right)+3\right)\right\}}=-1
\end{aligned}
$$

Using equation (2) we can obtain $c_{4}$ as

$$
\begin{aligned}
b_{4} c_{4}= & \frac{1}{2}-b_{2} c_{2}-b_{3} c_{3} \\
\Rightarrow c_{4} & =\frac{\frac{1}{2}-\left(\frac{3}{8} \times \frac{1}{3}\right)-\left(\frac{3}{8} \times \frac{2}{3}\right)}{\frac{1}{8}}=1
\end{aligned}
$$

Hence,

$$
\begin{aligned}
& c_{4}=a_{41}+a_{42}+a_{43} \\
& \Rightarrow a_{41}=c_{4}-a_{42}-a_{43}=1-(-1)-1=1 \\
& \text { Also } a_{32}=\frac{c_{3}\left(c_{2}-c_{3}\right)}{2 c_{2}\left(2 c_{2}-1\right)}=\frac{\frac{2}{3}\left(\frac{1}{3}-\frac{2}{3}\right)}{2 \times \frac{1}{3}\left(2 \times \frac{1}{3}-1\right)}=\frac{\frac{-2}{9}}{\frac{-2}{9}}=1
\end{aligned}
$$

From $c_{3}=a_{31}+a_{32}$

$\Rightarrow a_{31}=c_{3}-a_{32}=\frac{2}{3}-1=\frac{-1}{3}$

Finally, we know that $c_{1}=a_{11}=0$.

We have therefore determined all the unknowns in the method and the method can be written in Butcher's Tableu [3] as

\begin{tabular}{l|llll}
0 & 0 & 0 & 0 & 0 \\
$1 / 3$ & $1 / 3$ & 0 & 0 & 0 \\
$2 / 3$ & $-1 / 3$ & 1 & 0 & 0 \\
\hline & $1 / 8$ & $3 / 8$ & $3 / 8$ & $1 / 8$
\end{tabular}

Which has the form

$$
y_{n+1}=y_{n}+\frac{h}{8}\left(k_{1}+3 k_{2}+3 k_{3}+k_{4}\right)
$$




$$
\begin{aligned}
& k_{1}=f\left(x_{n}, y_{n}\right) \\
& k_{2}=f\left(x_{n}+c_{2} h, y_{n}+h a_{21} k_{1}\right)=f\left(x_{n}+\frac{h}{3}, y_{n}+\frac{h k_{1}}{3}\right) \\
& k_{3}=f\left(x_{n}+c_{3} h, y_{n}+h\left(a_{31} k_{1}+a_{32} k_{2}\right)\right)=f\left(x_{n}+\frac{2}{3} h, y_{n}+h\left(-\frac{1}{3} k_{1}+k_{2}\right)\right. \\
& k_{4}=\left(x_{n}+c_{4} h, y_{n}+h\left(a_{41} k_{1}+a_{42} k_{2}+a_{43} k_{3}\right)=f\left(x_{n}+h, y_{n}+h\left(k_{1}-k_{2}+k_{3}\right)\right.\right.
\end{aligned}
$$

\section{ANALYSIS OF THE METHOD}

The stability polynomial is given by

$R(h)=1+\bar{h} b^{T}(I-\bar{h} A)^{-1} e$ and it is required that $R(h)<1$ for absolute stability see [6]. Now for the Runge Kutta forth order method,

$$
y_{n+1}=y_{n}+\frac{h}{8}\left(k_{1}+3 k_{2}+3 k_{3}+k_{4}\right)
$$

The Butcher's Tableu is

$$
\begin{aligned}
& \begin{array}{l|llll}
0 & 0 & 0 & 0 & 0 \\
1 / 3 & 1 / 3 & 0 & 0 & 0 \\
2 / 3 & -1 / 3 & 1 & 0 & 0 \\
\hline 1 / 8 & 3 / 8 & 3 / 8 & 1 / 8 &
\end{array} \\
& A=\left(\begin{array}{cccc}
0 & 0 & 0 & 0 \\
\frac{1}{3} & 0 & 0 & 0 \\
-\frac{1}{3} & 1 & 0 & 0 \\
1 & -1 & 1 & 0
\end{array}\right), \quad I-\bar{h} A=\left(\begin{array}{cccc}
1 & 0 & 0 & 0 \\
\frac{-\bar{h}}{3} & 1 & 0 & 0 \\
\bar{h} & -\bar{h} & 1 & 0 \\
\overline{3} & \bar{h} & -\bar{h} & 1
\end{array}\right), \\
& \bar{h} b^{T}=\bar{h}\left(\begin{array}{llll}
\frac{1}{8} & \frac{3}{8} & \frac{3}{8} & \frac{1}{8}
\end{array}\right)=\left(\begin{array}{llll}
\frac{\bar{h}}{8} & \frac{3 \bar{h}}{8} & \frac{3 \bar{h}}{8} & \frac{\bar{h}}{8}
\end{array}\right) \\
& \therefore R(\bar{h})=1+\bar{h} b^{T}(I-\bar{h} A)^{-1} e \\
& =1+\left(\begin{array}{llll}
\frac{\bar{h}}{8} & \frac{3 \bar{h}}{8} & \frac{3 \bar{h}}{8} & \frac{\bar{h}}{8}
\end{array}\right)\left(\begin{array}{cccc}
1 & 0 & 0 & 0 \\
\frac{-\bar{h}}{3} & 1 & 0 & 0 \\
\frac{\bar{h}}{3} & -\bar{h} & 1 & 0 \\
-\bar{h} & \bar{h} & -\bar{h} & 1
\end{array}\right)^{-1}\left(\begin{array}{l}
1 \\
1 \\
1 \\
1
\end{array}\right)
\end{aligned}
$$

$$
\begin{aligned}
& =1+\left(\begin{array}{llll}
\frac{\bar{h}}{8} & \frac{3 \bar{h}}{8} & \frac{3 \bar{h}}{8} & \frac{\bar{h}}{8}
\end{array}\right)\left(\begin{array}{cccc}
1 & 0 & 0 & 0 \\
\frac{\bar{h}}{3} & 1 & 0 & 0 \\
\frac{-\bar{h}}{3}+\frac{\bar{h}^{2}}{3} & \bar{h} & 1 & 0 \\
\bar{h}-\frac{2 \bar{h}^{2}}{3}-\frac{\bar{h}^{3}}{3} & \bar{h}+\bar{h}^{2} & \bar{h} & 1
\end{array}\right)\left(\begin{array}{l}
1 \\
1 \\
1 \\
1
\end{array}\right) \\
& \left(\frac{\bar{h}}{8}+\frac{\bar{h}^{2}}{8}+\frac{3 \bar{h}}{8}\left(\frac{-\bar{h}}{3}+\frac{\bar{h}^{2}}{3}\right)+\frac{\bar{h}}{8}\left(\bar{h}-\frac{2 \bar{h}^{2}}{3}+\frac{\bar{h}^{3}}{3}\right)\right) \\
& =1+\quad \frac{3 \bar{h}}{8}+\frac{3 \bar{h}^{2}}{8}+\frac{\bar{h}}{8}\left(-\bar{h}+\bar{h}^{2}\right) \\
& \frac{3 \bar{h}}{8}+\frac{\bar{h}^{2}}{8} \\
& \frac{\bar{h}}{8} \\
& \left(\frac{\bar{h}}{8}+\frac{\bar{h}^{2}}{8}-\frac{3 \bar{h}^{2}}{24}+\frac{3 \bar{h}^{3}}{24}+\frac{\bar{h}^{2}}{8}-\frac{2 \bar{h}^{3}}{24}+\frac{\bar{h}^{4}}{24}\right) \\
& =1+\left(\begin{array}{c}
\frac{3 \bar{h}}{8}+\frac{3 \bar{h}^{2}}{8}-\frac{\bar{h}^{2}}{8}+\frac{\bar{h}^{3}}{8} \\
\frac{3 \bar{h}}{8}+\frac{\bar{h}^{2}}{8} \\
\frac{\bar{h}}{8}
\end{array}\right)\left(\begin{array}{l}
1 \\
1 \\
1 \\
1
\end{array}\right) \\
& =1+\frac{\bar{h}}{8}+\frac{\bar{h}^{2}}{8}+\frac{\bar{h}^{3}}{24}+\frac{\bar{h}^{4}}{24}+\frac{3 \bar{h}}{8}+\frac{\bar{h}^{2}}{4}+\frac{\bar{h}^{3}}{8}+\frac{3 \bar{h}}{8}+\frac{\bar{h}^{2}}{8}+\frac{\bar{h}}{8} \\
& =1+\bar{h}+\frac{\bar{h}^{2}}{2}+\frac{\bar{h}^{3}}{6}+\frac{\bar{h}^{4}}{24}
\end{aligned}
$$

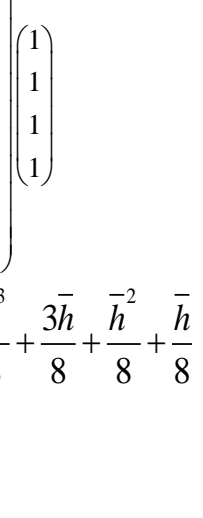

For absolute stability

$$
-1<\left|1+\bar{h}+\frac{1}{2} \bar{h}^{2}+\frac{1}{6} \bar{h}^{3}+\frac{1}{24} \bar{h}^{4}\right|<1
$$

Taking the RHS

$\left|1+\bar{h}+\frac{1}{2} \bar{h}^{2}+\frac{1}{6} \bar{h}^{3}+\frac{1}{24} \bar{h}^{4}\right|<1$

$\bar{h}+\frac{1}{2} \bar{h}^{2}+\frac{1}{6} \bar{h}^{3}+\frac{1}{24} \bar{h}^{4}<0$

Using Mathematica we get the roots as

NSolve[h+h*h/2+h*h*h/6+h*h*h*h/24==0,h]

$\{\{\mathrm{h} \square-2.78529\},\{\mathrm{h} \square-0.607353-2.8719$

$\square\},\{\mathrm{h} \square$

$0.607353+2.8719 \square\},\{\mathrm{h} \square 0\}$.

We consider 3 cases as it can be found in [1]

Case 1

When $\lambda$ is real and $\lambda<0$,

The roots are -2.785 and 0 
Hence the stability interval is $h \in(-2.785,0)$.

\section{Case 2}

$=1+\left(\begin{array}{c}\frac{\bar{h}}{8}+\frac{\bar{h}^{2}}{8}+\frac{\bar{h}^{3}}{24}+\frac{\bar{h}^{4}}{24} \\ \frac{3 \bar{h}}{8}+\frac{\bar{h}^{2}}{4}+\frac{\bar{h}^{3}}{8} \\ \frac{3 \bar{h}}{8}+\frac{\bar{h}^{2}}{8} \\ \frac{\bar{h}}{8}\end{array}\right)\left(\begin{array}{l}1 \\ 1 \\ 1 \\ 1\end{array}\right)$

When $\lambda h$ is pure and

imaginary,

We set $\lambda=i y$ in the stability polynomial to get

$$
\begin{aligned}
& \left|1+i(y h)-\frac{(y h)^{2}}{2}-i \frac{(y h)^{3}}{6}+\frac{(y h)^{4}}{24}\right|<1 \\
& \Rightarrow\left|1-\frac{(y h)^{2}}{2}+\frac{(y h)^{4}}{24}+(i y h)-i \frac{(y h)^{3}}{6}\right|<1
\end{aligned}
$$

Let $t=y h$ and take the magnitude

$$
\begin{aligned}
& \Rightarrow\left(1-\frac{t^{2}}{2}+\frac{t^{4}}{24}\right)^{2}+\left(t-\frac{t^{3}}{6}\right)<1 \\
& \left(1-\frac{t^{2}}{2}+\frac{t^{4}}{24}-\frac{t^{2}}{2}+\frac{t^{4}}{4}-\frac{t^{6}}{48}+\frac{t^{4}}{24}-\frac{t^{6}}{48}+\frac{t^{8}}{578}\right)+\left(t^{2}-\frac{t^{4}}{6}-\frac{t^{4}}{6}+\frac{t^{6}}{36}\right)<1
\end{aligned}
$$

Simplifying, we get

$$
\begin{aligned}
& 1-\frac{t^{6}}{72}+\frac{t^{8}}{576}<1 \\
& \Rightarrow \frac{t^{6}}{72}+\frac{t^{8}}{576}<0
\end{aligned}
$$

Using Mathematica to find the roots we have

NSolve $\left[\left(-\left(t^{\wedge} 6\right) / 72\right)+\left(\left(t^{\wedge} 8\right) / 576\right) \square 0, t\right]$

$\{\{t \rightarrow-$

$.82843\},\{t \rightarrow 0\},.\{t \rightarrow 0\},.\{t \rightarrow 0\},.\{t \rightarrow 0\},.\{t \rightarrow 0\},\{$.

$t \rightarrow 0.\},\{t \rightarrow 2.82843\}\}$

The equation is satisfied for

$|t|<2.82843$

i.e. $|t|<2 \sqrt{2}$

Hence the stability interval is $0<\bar{h}<2 \sqrt{2}$. i.e. $\bar{h} \in(0,2 \sqrt{2})$

Case 3 When $\lambda$ is complex with $\operatorname{Re}(\lambda)>0$, we set $\lambda=x+i y$ in $\left|1+\lambda h+\frac{(\lambda h)^{2}}{2}+\frac{(\lambda h)^{3}}{6}+\frac{(\lambda h)^{4}}{24}\right|<1$

and plot the boundary of the region by plotting the real and imaginary parts.

The stability region is plotted using Maple as follows

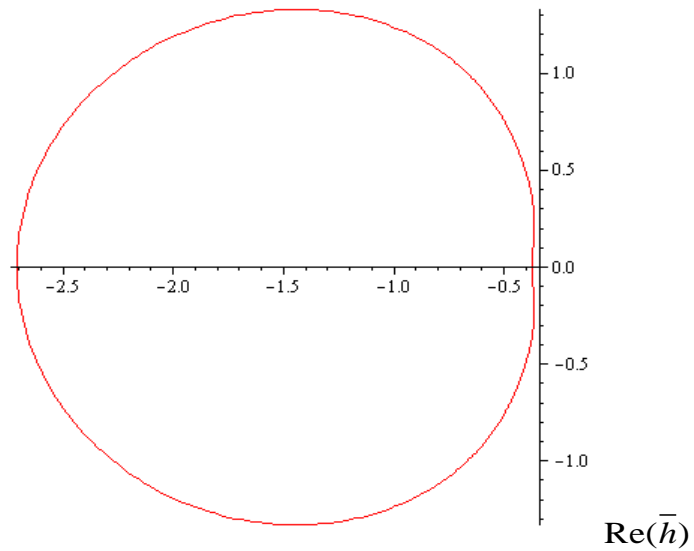

\section{CONCLUSION}

In this paper, we have simplified the existing derivation and analysis of the fourth order Runge-Kutta Method for easy reference to students and plot the stability region. We also reduced the complexity of the method by proposing a step by step derivation approach for better understanding to students.

\section{REFERENCES}

[1] M.K. Jain, S.R.K. Iyengar, R.K. Jain, (2007), Numerical Methods for Scientific and Engineering Computing.

[2] J. D. Lambert, (1991), Numerical Methods for Ordinary Differential Systems, the initial value Problem, John Wiley \& Sons Ltd.

[3] J.C. Butcher, (2003), Numerical Methods for Ordinary Differential Equations, John Wiley \& Sons Ltd.

[4] John R. Dorman, (1996), Numerical Methods for Differential Equations, a Computational Approach, CRC Press, Inc.

[5] J. D. Lambert, (1973), Computational Methods in Ordinary Differential Equations, John Wiley \& Sons Ltd

[6] Fudzia Ismail, (2010), Lecture Notes on Numerical Methods (unpublished), University Putra Malaysia

[7] G. Byrne and Hindmarsh, (1990), RK Methods prove popular at IMA Conference on Numerical ODE's,SIAM News, $23 / 2$ pp.14-15.

[8] Lawrence F. Shampine, (1985),Interpolation for RungeKutta Methods.SIAM Journal of numerical analysis,22/5,pp.1014-1027. 